\title{
Distributional curvature of time dependent cosmic strings
}

\author{
J. P. Wilson
}

Department of Mathematics, University of Southampton, Southampton, SO17 1BJ

\begin{abstract}
Colombeau's theory of generalised functions is used to calculate the contributions, at the rotation axis, to the distributional curvature for a time-dependent radiating cosmic string, and hence the mass per unit length of the string source. This mass per unit length is compared with the mass at null infinity, giving evidence for a global energy conservation law.
\end{abstract}

\section{Introduction}

An important problem in relativity is the calculation of distributional curvatures of non-regular metrics. Such curvatures represent physical fields whose support is confined to a proper submanifold of space-time, such as the point mass in the Schwarzschild solution and cosmic strings.

The main obstacle to rigorously calculating such curvatures is the ambiguity that arises when one tries to multiply distributions, because the Riemann tensor is a nonlinear function of the metric and its derivatives. It was proved by Schwartz (1954) that one cannot define an associative multiplication of distributions which is compatible with the usual operation of differentiation. There are metrics for which the distributional Riemann tensor may be calculated directly without the need to multiply distributions, but this class of metrics is very small. In fact a necessary condition for the metric to lie in this class is that the distributional curvature must have its support on a submanifold of codimension of at most one (Geroch and Traschen, 1987); thus it is possible for metrics representing matter shells to lie in this class, but not for strings and point particles.

In an earlier paper (Clarke et al 1996) it was shown how one may apply Colombeau's theory of Generalised Functions (Colombeau, 1983, 1990), in which nonlinear operations are well defined, to the calculation of distributional curvatures where a direct calculations would not work. In Colombeau's theory, the Schwartzian space of distributions $\mathcal{D}^{\prime}\left(\mathbf{R}^{n}\right)$ is embedded as a linear subspace of a much larger differential algebra $\mathcal{G}\left(\mathbf{R}^{n}\right)$ by the smoothing operation

$$
\tilde{f}(\Phi, \mathbf{x})=\frac{1}{\varepsilon^{n}} \int_{\mathbf{R}^{n}} f\left(\mathbf{x}^{\prime}\right) \Phi\left(\frac{\mathbf{x}^{\prime}-\mathbf{x}}{\varepsilon}\right) \mathrm{d} x^{\prime},
$$

where $\Phi \in \mathcal{D}\left(\mathbf{R}^{n}\right)$ has unit radius. Thus products of two distributions are defined in this algebra, although one cannot interpret this product as a distribution via the embedding. However one may give a distributional interpretation to this object via the notion of weak equivalence of two generalised functions $F$ and $G$ (written as $F \approx G$ ) which essentially is the generalisation of the equality of two distributions to $\mathcal{G}\left(\mathbf{R}^{n}\right)$.

In the previous paper (Clarke et al 1996), the focus was on calculating distributional curvatures of 2 -surfaces, in particular the cone with a metric of

$$
\mathrm{d} s^{2}=\mathrm{d} r^{2}+A^{2} r^{2} \mathrm{~d} \phi^{2}, \quad|A|<1 .
$$

Here the Gaussian curvature density $\frac{1}{2} R \sqrt{g}$ (as a generalised function) is weakly equivalent to the distribution $2 \pi(1-A) \delta^{(2)}(x, y)$, where $(x, y)=(r \cos \phi, r, \sin \phi)$. In this paper we shall consider applications to axisymmetric dynamical space-times with conical singularities and in particular space-times with a pure radiation energy-momentum tensor (Kramer et al 1980). That is with the energy-momentum tensor of the form

$$
T_{a b}=\Phi^{2} k_{a} k_{b}, \quad k_{a} k^{a}=0,
$$

where vector $k^{a}$ is the null direction of propagation and $\Phi$ represents the amplitude. In the case of cylindrical symmetry one may write the metric as

$$
d s^{2}=e^{-2 \psi}\left(e^{2 \gamma}\left(-d t^{2}+d r^{2}\right)+r^{2} d \phi^{2}\right)+e^{2 \psi} d z^{2},
$$


where $\psi$ and $\gamma$ are functions of $t$ and $r$. One may then obtain a pure radiation solution with

$$
\begin{aligned}
& k_{t}=-k_{r}=1 \\
& \Phi^{2}=r^{-1} \gamma_{, r}-\psi_{, t}{ }^{2}-\psi_{, r}{ }^{2}
\end{aligned}
$$

by solving the following field equations (Kramer et al 1980; Krishna Rao, 1964)

$$
\begin{aligned}
& \psi_{, r r}+r^{-1} \psi_{, r}-\psi_{, t t}=0 \\
& \gamma_{, r}+\gamma_{, t}=r\left(\psi_{, r}+\psi_{, t}\right)^{2}
\end{aligned}
$$

The Colombeau framework will be used to extract the distributional contributions at the axis $r=0$ for the Riemann tensor densities $R^{a b}{ }_{c d} \sqrt{-g}$ and the corresponding distributional contributions for the energy-momentum tensor, which is defined via the field equations

$$
R_{a b}-\frac{1}{2} R g_{a b}=T_{a b}
$$

Such distributional contributions may then be used to give a definition of mass per unit length of such a string.

Finally we shall consider the physical implications; the behaviour of matter at null infinity, using a three dimensional asymptotic formalism, developed be Ashtekar et al (1997). This will Demonstrate the sense in which global conservation holds.

\section{Distributional curvature of pure radiation fields}

In this paper we shall focus on the distributional curvature of a particular cylindrically symmetrical pure radiation solution $(\psi=0, \gamma=\gamma(t-r))$, where $\gamma(u)$ is an arbitrary $C^{\infty}$ function, whose metric may be expressed as

$$
\mathrm{d} s^{2}=\mathrm{e}^{2 \gamma(t-r)}\left(-\mathrm{d} t^{2}+\mathrm{d} r^{2}\right)+r^{2} \mathrm{~d} \phi^{2}+\mathrm{d} z^{2} .
$$

We may write this metric in null Cartesian coordinates $(u=t-r, x=r \cos \phi, y=r \sin \phi, z)$;

$$
\begin{aligned}
\mathrm{d} s^{2}= & -\mathrm{e}^{2 \gamma(u)} \mathrm{d} u^{2}+\frac{1}{2}\left(\mathrm{~d} x^{2}+\mathrm{d} y^{2}\right)+\mathrm{d} z^{2}-2 \mathrm{e}^{2 \gamma(u)}\left(\frac{x}{r} \mathrm{~d} u \mathrm{~d} x+\frac{y}{r} \mathrm{~d} u \mathrm{~d} y\right) \\
& -\frac{1}{2}\left(\frac{x^{2}-y^{2}}{r^{2}} \mathrm{~d} x^{2}+4 \frac{x y}{r^{2}} \mathrm{~d} x \mathrm{~d} y+\frac{y^{2}-x^{2}}{r^{2}} \mathrm{~d} y^{2}\right) .
\end{aligned}
$$

Except at the singularity $r=0$, the Riemann curvature density will have non-zero independent components

$$
R^{x y}{ }_{u x} \sqrt{-g}=-\gamma^{\prime} / r \sin \phi, \quad R^{x y}{ }_{u y} \sqrt{-g}=\gamma^{\prime} / r \cos \phi .
$$

which may of course be interpreted as distributions since such functions will always be locally integrable over $\mathbf{R}^{2}$. This does not however mean that this distributional curvature will be valid at the axis; we could expect a delta function contribution to the distributional curvature due to a non-zero angular deficit which may be shown to be

$$
\lim _{r \rightarrow 0} 2 \pi\left\{1-\left(\frac{g_{\phi \phi ; a} g_{\phi \phi} ; a}{4 g_{\phi \phi}}\right)^{1 / 2}\right\}=2 \pi\left(1-e^{-\gamma(t)}\right),
$$

as in the case of the static cone (See Clarke et al 1996). We shall be using a regularisation based upon Colombeau's generalised functions to recover the distributional contributions, on the axis, to the curvature.

It may be remarked that this singularity is not quasi-regular but intermediate; that is the Riemann tensor components do not converge in the parallelly propagated orthonormal frame $\mathbf{E}$ along the integral curves of $\partial / \partial r$

$$
\begin{aligned}
& \mathbf{E}_{0}=\frac{1}{2}\left(1+\mathrm{e}^{-2 \gamma}\right) \frac{\partial}{\partial t}+\frac{1}{2}\left(1-\mathrm{e}^{-2 \gamma}\right) \frac{\partial}{\partial r} \\
& \mathbf{E}_{1}=\frac{1}{2}\left(1-\mathrm{e}^{-2 \gamma}\right) \frac{\partial}{\partial t}+\frac{1}{2}\left(1+\mathrm{e}^{-2 \gamma}\right) \frac{\partial}{\partial r} \\
& \mathbf{E}_{2}=r^{-1} \frac{\partial}{\partial \phi} \\
& \mathbf{E}_{3}=\frac{\partial}{\partial z}
\end{aligned}
$$


in which

$$
R_{0202}=-R_{0212}=R_{1212}=-\mathrm{e}^{-4 \gamma} \frac{\gamma^{\prime}}{r} .
$$

However there is an orthonormal frame $\mathbf{E}^{\prime}$ in which they do converge

$$
\begin{aligned}
& \mathbf{E}_{0}^{\prime}=\frac{1+r^{2}}{2 r} \mathrm{e}^{-\gamma} \frac{\partial}{\partial t}+\frac{1-r^{2}}{2 r} \mathrm{e}^{-\gamma} \frac{\partial}{\partial r} \\
& \mathbf{E}_{1}^{\prime}=\frac{1-r^{2}}{2 r} \mathrm{e}^{-\gamma} \frac{\partial}{\partial t}+\frac{1+r^{2}}{2 r} \mathrm{e}^{-\gamma} \frac{\partial}{\partial r} \\
& \mathbf{E}_{2}^{\prime}=r^{-1} \frac{\partial}{\partial \phi} \\
& \mathbf{E}_{3}^{\prime}=\frac{\partial}{\partial z}
\end{aligned}
$$

for which

$$
R_{0202}^{\prime}=-R_{0212}^{\prime}=R_{1212}^{\prime}=-r \mathrm{e}^{-2 \gamma} \gamma^{\prime}
$$

We shall expect our distributional curvature to have cylindrical symmetry; this suggests that we should calculate $\widetilde{g_{a b}}$ by smoothing kernel $\Phi(u, x, y, z) \in \mathcal{A}_{1}\left(\mathbf{R}^{4}\right)$, with radius

$$
R_{0}=\sup \left\{\left(x^{2}+y^{2}\right)^{1 / 2}\left|\int_{\mathbf{R}^{2}}\right| \Phi(u, x, y, z) \mid \mathrm{d} u \mathrm{~d} z>0\right\}
$$

The smoothed metric may then be written as

$$
\widetilde{g_{a b}}=\frac{1}{\varepsilon^{4}} \int_{\mathbf{R}^{4}} g_{a b}\left(u^{\prime}+u, x^{\prime}+x, y^{\prime}+y, z^{\prime}+z\right) \Phi\left(u^{\prime} / \varepsilon, x^{\prime} / \varepsilon, y^{\prime} / \varepsilon, z^{\prime} / \varepsilon\right) \mathrm{d} u^{\prime} \mathrm{d} x^{\prime} \mathrm{d} y^{\prime} \mathrm{d} z^{\prime}
$$

We shall expect our distributional curvature to have cylindrical symmetry; this suggests that it should be sufficient to use a cylindrically symmetrical smoothing kernel, which we shall denote as

$$
\Phi(u, r)=\Phi(u, r \cos \phi, r \sin \phi, z)
$$

With such a kernel the smoothed metric becomes

$$
\widetilde{g_{a b}}=\frac{1}{\varepsilon^{3}} \int_{\mathbf{R}^{4}} g_{a b}\left(u^{\prime}+u, x^{\prime}+x, y^{\prime}+y\right) \Phi\left(u^{\prime} / \varepsilon,\left(x^{\prime 2}+y^{\prime 2}\right)^{1 / 2} / \varepsilon\right) \mathrm{d} u^{\prime} \mathrm{d} x^{\prime} \mathrm{d} y^{\prime} .
$$

The metric components will be in general sums of $C^{\infty}$ terms, which may be identified with their smoothings in $\mathcal{E}_{M}\left(\mathbf{R}^{4}\right)$, and singular components. The only functions that we will have to smooth are

(i) $(u, x, y, z) \mapsto \mathrm{e}^{2 \mathrm{i} \phi}$

(ii) $(u, x, y, z) \mapsto \mathrm{e}^{2 \gamma(u)+\mathrm{i} \phi}$.

For (i) the smoothing is given by (see Clarke et al 1996)

$$
\begin{aligned}
\widetilde{\mathrm{e}^{2 \mathrm{i} \phi}} & =\frac{1}{\varepsilon^{3}} \int_{-\infty}^{\infty} \int_{0}^{\infty} \int_{0}^{2 \pi}\left(\frac{r \mathrm{e}^{\mathrm{i} \phi}+r^{\prime} \mathrm{e}^{\mathrm{i} \phi^{\prime}}}{r \mathrm{e}^{\mathrm{i} \phi^{\prime}}+r^{\prime} \mathrm{e}^{\mathrm{i} \phi}}\right) \mathrm{e}^{\mathrm{i}\left(\phi+\phi^{\prime}\right)} \Phi\left(u^{\prime} / \varepsilon, r^{\prime} / \varepsilon\right) r^{\prime} \mathrm{d} u^{\prime} \mathrm{d} r^{\prime} \mathrm{d} \phi^{\prime} \\
& =H_{\varepsilon}(r) \mathrm{e}^{2 i \phi}
\end{aligned}
$$

where

$$
H_{\varepsilon}(r)= \begin{cases}2 \pi \int_{-\infty}^{\infty} \int_{0}^{r / \varepsilon}\left(1-\frac{\varepsilon^{2} r^{\prime 2}}{r^{2}}\right) \Phi\left(u^{\prime}, r^{\prime}\right) r^{\prime} \mathrm{d} u^{\prime} \mathrm{d} r^{\prime}, & \text { for } r<\varepsilon \\ 1-2 \pi \frac{\varepsilon^{2}}{r^{2}} \int_{-\infty}^{\infty} \int_{0}^{R_{0}} r^{\prime 3} \Phi\left(u^{\prime}, r^{\prime}\right) \mathrm{d} u^{\prime} \mathrm{d} r^{\prime}, & \text { for } r>\varepsilon\end{cases}
$$


For (ii) the smoothing is given by

$$
\mathrm{e}^{2 \gamma(u)+\mathrm{i} \phi}=\frac{1}{\varepsilon^{3}} \int_{-\infty}^{\infty} \int_{0}^{\infty} \int_{0}^{2 \pi} e^{2 \gamma\left(u+u^{\prime}\right)}\left(\frac{r \mathrm{e}^{\mathrm{i} \phi}+r^{\prime} \mathrm{e}^{\mathrm{i} \phi^{\prime}}}{r \mathrm{e}^{\mathrm{i} \phi^{\prime}}+r^{\prime} \mathrm{e}^{\mathrm{i} \phi}}\right)^{1 / 2} \mathrm{e}^{\mathrm{i}\left(\phi+\phi^{\prime}\right) / 2} \Phi\left(u^{\prime} / \varepsilon, r^{\prime} / \varepsilon\right) r^{\prime} \mathrm{d} u^{\prime} \mathrm{d} r^{\prime} \mathrm{d} \phi^{\prime}
$$

in which $\phi^{\prime}$ may be integrated out by complex contour integration; If

$$
I=\int_{0}^{2 \pi}\left(\frac{r \mathrm{e}^{\mathrm{i} \phi}+r^{\prime} \mathrm{e}^{\mathrm{i} \phi^{\prime}}}{r \mathrm{e}^{\mathrm{i} \phi^{\prime}}+r^{\prime} \mathrm{e}^{\mathrm{i} \phi}}\right)^{1 / 2} \mathrm{e}^{\mathrm{i}\left(\phi+\phi^{\prime}\right) / 2} \mathrm{~d} \phi^{\prime},
$$

then setting $w=\mathrm{e}^{\mathrm{i} \phi}$ and $z=\mathrm{e}^{\mathrm{i} \phi^{\prime}}$, we may integrate out $\phi^{\prime}$ by integrating the complex function

$$
\Gamma(z)=-\mathrm{i}\left(\frac{r^{\prime} z+r w}{r z+r^{\prime} w}\right)^{1 / 2} \frac{w}{z}^{1 / 2}
$$

around the circular contour $\kappa:|z|=1$ to obtain

$$
I= \begin{cases}4 E\left(r^{\prime} / r\right) e^{i \phi}, & \text { for } r^{\prime}<r \\ 4 G\left(r / r^{\prime}\right) e^{i \phi}, & \text { for } r^{\prime}>r\end{cases}
$$

where the hypergeometric functions $E(\lambda)$ and $G(\lambda)$ are defined by

$$
\begin{aligned}
& E(s)=\int_{0}^{1}\left(\frac{1-\lambda^{2} s^{2}}{1-\lambda^{2}}\right)^{1 / 2} \mathrm{~d} \lambda \\
& G(s)=s \int_{0}^{1}\left(\frac{1-\lambda^{2}}{1-\lambda^{2} s^{2}}\right)^{1 / 2} \mathrm{~d} \lambda
\end{aligned}
$$

This enables us to express $\mathrm{e}^{2 \gamma(u)+\mathrm{i} \phi}$ as $L_{\varepsilon}(u, r) \mathrm{e}^{2 \gamma(u)+\mathrm{i} \phi}$, where

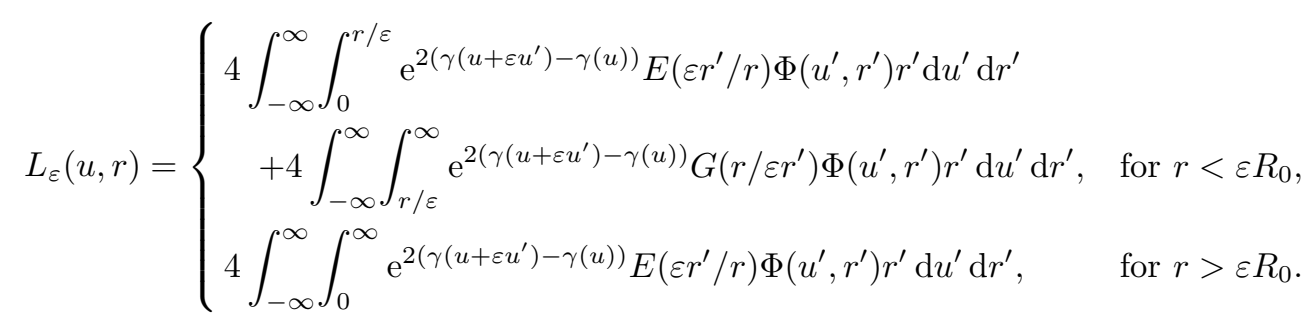

We may therefore express $\widetilde{g_{a b_{\varepsilon}}}$ as

$$
\begin{aligned}
\widetilde{\mathrm{d} s^{2}}= & -\mathrm{e}^{2 \gamma(u)} \mathrm{d} u^{2}+\frac{1}{2}\left(\mathrm{~d} x^{2}+\mathrm{d} y^{2}\right)+\mathrm{d} z^{2}-2 \mathrm{e}^{2 \gamma(u)} L_{\varepsilon}(u, r)\left(\frac{x}{r} \mathrm{~d} u \mathrm{~d} x+\frac{y}{r} \mathrm{~d} u \mathrm{~d} y\right) \\
& -\frac{1}{2} H_{\varepsilon}(r)\left(\frac{x^{2}-y^{2}}{r^{2}} \mathrm{~d} x^{2}+4 \frac{x y}{r^{2}} \mathrm{~d} x \mathrm{~d} y+\frac{y^{2}-x^{2}}{r^{2}} \mathrm{~d} y^{2}\right) .
\end{aligned}
$$

The smoothed metric will be used to calculate distributional curvatures. In almost all cases, the following estimates for $H_{\varepsilon}(r)$ and $L_{\varepsilon}(u, r)$ will prove to be sufficient;

$$
\begin{gathered}
H_{\varepsilon}(r)= \begin{cases}O\left(r^{2} / \varepsilon^{2}\right), & \text { for } r<\varepsilon R_{0}, \\
1+O\left(\varepsilon^{2} / r^{2}\right), & \text { for } r>\varepsilon R_{0}\end{cases} \\
L_{\varepsilon}(u, r)= \begin{cases}O\left(r^{2} / \varepsilon^{2}\right), & \text { for } r<\varepsilon R_{0}, \\
1+O\left(\varepsilon^{2} / r^{2}\right), & \text { for } r>\varepsilon R_{0}\end{cases}
\end{gathered}
$$

It must be emphasised that $L_{\varepsilon}$ retains its dependence of $u$, even though we shall omit it from the order notation; on the other hand, $H_{\varepsilon}$ is only dependent on $r$ and $\varepsilon$. 
We now calculate the generalised Riemann density functions $\tilde{R}_{c d}^{a b} \sqrt{-\tilde{g}}$ from the smoothed metric $\widetilde{g_{a b}} \varepsilon$

$$
\begin{aligned}
& \tilde{R}_{u x \varepsilon}^{u x} \sqrt{-\tilde{g}_{\varepsilon}}=P_{0}+P_{1} \cos 2 \phi \quad \tilde{R}^{u y}{ }_{u y_{\varepsilon}} \sqrt{-\tilde{g}_{\varepsilon}}=P_{0}-P_{1} \cos 2 \phi \\
& \tilde{R}_{u y_{\varepsilon}}^{u x} \sqrt{-\tilde{g}_{\varepsilon}}=\tilde{R}_{u x \varepsilon}^{u y} \sqrt{-\tilde{g}_{\varepsilon}}=P_{1} \sin 2 \phi \\
& \tilde{R}^{x y}{ }_{u x \varepsilon} \sqrt{-\tilde{g}_{\varepsilon}}=-P_{2} \sin \phi \quad \tilde{R}^{x y}{ }_{u y_{\varepsilon}} \sqrt{-\tilde{g}_{\varepsilon}}=P_{2} \cos \phi \\
& \tilde{R}_{x y_{\varepsilon}}^{u x} \sqrt{-\tilde{g}_{\varepsilon}}=L_{\varepsilon} P_{3} \sin \phi \quad \tilde{R}_{x y_{\varepsilon}}^{u y} \sqrt{-\tilde{g}_{\varepsilon}}=-L_{\varepsilon} P_{3} \cos \phi \\
& \tilde{R}^{x y}{ }_{x y_{\varepsilon}} \sqrt{-\tilde{g}_{\varepsilon}}=P_{3}
\end{aligned}
$$

where

$$
\begin{aligned}
& P_{0}= \begin{cases}O\left(r / \varepsilon^{2}\right), & \text { for } r<\varepsilon R_{0}, \\
O\left(\varepsilon^{2} / r^{3}\right), & \text { for } r>\varepsilon R_{0},\end{cases} \\
& P_{1}= \begin{cases}O\left(r / \varepsilon^{2}\right), & \text { for } r<\varepsilon R_{0}, \\
O\left(\varepsilon^{2} / r^{3}\right), & \text { for } r>\varepsilon R_{0},\end{cases} \\
& P_{2}= \begin{cases}-\gamma(u) / r+O(1 / r), & \text { for } r<\varepsilon R_{0}, \\
-\gamma(u) / r+O\left(\varepsilon^{2} / r^{3}\right), & \text { for } r>\varepsilon R_{0},\end{cases} \\
& P_{3}= \begin{cases}O\left(1 / \varepsilon^{2}\right), & \text { for } r<\varepsilon R_{0}, \\
O\left(\varepsilon^{2} / r^{4}\right), & \text { for } r>\varepsilon R_{0},\end{cases}
\end{aligned}
$$

To obtain the distributional parts to the curvature on the axis not contained in the expressions for $R^{a b}{ }_{c d} \sqrt{-g}$, one must evaluate the limits

$$
\lim _{\varepsilon \rightarrow 0} \int_{\mathbf{R}^{4}}\left(\tilde{R}_{c d \varepsilon}^{a b} \sqrt{-\tilde{g}_{\varepsilon}}-R_{c d}^{a b} \sqrt{-g}\right) \Psi \mathrm{d} u \mathrm{~d} x \mathrm{~d} y \mathrm{~d} z
$$

where $\Psi$ is an arbitrary function in $\mathcal{D}\left(\mathbf{R}^{4}\right)$. The following notations will be used throughout the analysis;

$$
\begin{aligned}
& K=\operatorname{supp} \Psi \\
& K^{\prime}=\{(u, z) \mid(u, x, y, z) \in K\}, \\
& B_{\varepsilon}=\left\{(u, x, y, z) \in K \mid\left(x^{2}+y^{2}\right)^{1 / 2} \leq \varepsilon R_{0}\right\}, \\
& R_{K}=\sup \left\{\left(x^{2}+y^{2}\right)^{1 / 2} \mid(u, x, y, z) \in K\right\} .
\end{aligned}
$$

We shall first show that $\left[\tilde{R}^{x y} u x \varepsilon \sqrt{-\tilde{g}_{\varepsilon}}-R^{x y} u x \sqrt{-g}\right] \approx 0$. That is

$$
\int_{\mathbf{R}^{4}}\left(\tilde{R}_{u x \varepsilon}^{x y} \sqrt{-\tilde{g}_{\varepsilon}}-R^{x y}{ }_{u x} \sqrt{-g}\right) \Psi(u, x, y, z) \mathrm{d} u \mathrm{~d} x \mathrm{~d} y \mathrm{~d} z \rightarrow 0 .
$$

We have

$$
\begin{aligned}
& \left|\int_{\mathbf{R}^{4}}\left(\tilde{R}_{u x \varepsilon}^{x y} \sqrt{-\tilde{g}_{\varepsilon}}-R_{u x}^{x y} \sqrt{-g}\right) \Psi \mathrm{d} u \mathrm{~d} x \mathrm{~d} y \mathrm{~d} z\right| \\
& \leq M_{1} \int_{B_{\varepsilon}}\left|\tilde{R}_{u x \varepsilon}^{x y} \sqrt{-\tilde{g}_{\varepsilon}}-R^{x y} u x \sqrt{-g}\right| \mathrm{d} u \mathrm{~d} x \mathrm{~d} y \mathrm{~d} z \\
& +M_{1} \int_{K-B_{\varepsilon}}\left|\tilde{R}^{x y} u x-\sqrt{-\tilde{g}_{-}} R^{x y} u x \sqrt{-g}\right| \mathrm{d} u \mathrm{~d} x \mathrm{~d} y \mathrm{~d} z \\
& \leq M_{2} \varepsilon+M_{3}\left(\frac{\varepsilon^{2}}{\varepsilon R_{0}}-\frac{\varepsilon^{2}}{R_{K}}\right)
\end{aligned}
$$

where we shall use $M_{i}$ to denote positive constants. Therefore

$$
\int_{\mathbf{R}^{4}}\left(\tilde{R}_{u x \varepsilon}^{x y} \sqrt{-\tilde{g}_{\varepsilon}}-R_{u x}^{x y} \sqrt{-g}\right) \Psi(u, x, y, z) \mathrm{d} u \mathrm{~d} x \mathrm{~d} y \mathrm{~d} z=O(\varepsilon) .
$$


Similarly it may be shown that

$$
\begin{aligned}
& {\left[\tilde{R}^{x y}{ }_{u \alpha} \sqrt{-\tilde{g}}\right] \approx R^{x y}{ }_{u \alpha} \sqrt{-g}} \\
& {\left[\tilde{R}^{u \alpha}{ }_{u \beta} \sqrt{-\tilde{g}}\right] \approx 0 .}
\end{aligned}
$$

For the component $\tilde{R}_{x y}^{u x} \sqrt{-\tilde{g}}$ (and similarly for $\tilde{R}_{x y}^{u y} \sqrt{-\tilde{g}}$ ), we may write (using the mean value theorem)

$$
\int_{\mathbf{R}^{4}} \tilde{R}_{x y_{\varepsilon}}^{u x} \sqrt{-\tilde{g}_{\varepsilon}} \Psi \mathrm{d} u \mathrm{~d} x \mathrm{~d} y \mathrm{~d} z=I_{1}+I_{2}
$$

where

$$
\begin{aligned}
I_{1} & =\int_{K} \tilde{R}_{x y_{\varepsilon}}^{u x} \sqrt{-\tilde{g}_{\varepsilon}} \Psi(u, 0,0, z) \mathrm{d} u \mathrm{~d} x \mathrm{~d} y \mathrm{~d} z \\
I_{2} & =\int_{K} \tilde{R}_{x y_{\varepsilon}}^{u x} \sqrt{-\tilde{g}_{\varepsilon}} \frac{\mathrm{d} \Psi}{\mathrm{d} r}(u, \xi x, \xi y, z) r \mathrm{~d} u \mathrm{~d} x \mathrm{~d} y \mathrm{~d} z \\
\xi & \in[0,1]
\end{aligned}
$$

now

$$
\begin{aligned}
\left|I_{2}\right| \leq & M_{4} \int_{B_{\varepsilon}}\left|\tilde{R}_{x y_{\varepsilon}}^{u x} \sqrt{-\tilde{g}_{\varepsilon}}\right| r \mathrm{~d} u \mathrm{~d} x \mathrm{~d} y \mathrm{~d} z \\
& +M_{4} \int_{K-B_{\varepsilon}}\left|\tilde{R}_{x y_{\varepsilon}}^{u x} \sqrt{-\tilde{g}_{\varepsilon}}\right| r \mathrm{~d} u \mathrm{~d} x \mathrm{~d} y \mathrm{~d} z \\
\leq & M_{5} \frac{\left(\varepsilon R_{0}\right)^{3}}{\varepsilon^{2}}+M_{6} \varepsilon^{2}\left(\frac{1}{\varepsilon R_{0}}-\frac{1}{R_{K}}\right)
\end{aligned}
$$

and

$$
I_{1}=\int_{K^{\prime}}\left(\int_{0}^{\infty} L_{\varepsilon} P_{3} r \mathrm{~d} r\right)\left(\int_{0}^{2 \pi} \sin \phi \mathrm{d} \phi\right) \Psi(u, 0,0, z) \mathrm{d} u d z
$$

which vanishes because our radial integral has no $\phi$-dependence and is bounded. This will imply that

$$
\int_{\mathbf{R}^{4}} \tilde{R}_{x y_{\varepsilon}}^{u x} \sqrt{-\tilde{g}_{\varepsilon}} \Psi \mathrm{d} u \mathrm{~d} x \mathrm{~d} y \mathrm{~d} z=O(\varepsilon)
$$

and hence that

$$
\tilde{R}_{x y}^{u \alpha} \sqrt{-\tilde{g}} \approx 0 \quad \alpha=x, y
$$

Only one component now remains; $\tilde{R}_{x y}^{x y} \sqrt{-\tilde{g}}$, which we can expect to have a delta function contribution to the distributional curvature at the axis. We shall require a more accurate estimate for $\tilde{R}_{x y \varepsilon} \sqrt{-\tilde{g}_{\varepsilon}}$ derived from

$$
\tilde{R}_{x y_{\varepsilon}}^{x y} \sqrt{-\tilde{g}_{\varepsilon}}=-\mathrm{e}^{\gamma} \frac{\mathrm{d}}{\mathrm{d} r}\left[\frac{2 \pi \int_{0}^{r / \varepsilon} \Phi\left(r^{\prime}\right) r^{\prime} \mathrm{d} r+1}{\left(1+H_{\varepsilon}\right)^{1 / 2}\left(1-H_{\varepsilon}+2 \mathrm{e}^{2 \gamma} L_{\varepsilon}^{2}\right)^{2}}\right]
$$

This will imply that

$$
\int_{0}^{R_{K}} \tilde{R}_{x y_{\varepsilon}}^{x y} \sqrt{-\tilde{g}_{\varepsilon}} r \mathrm{~d} r=\mathrm{e}^{\gamma}\left(1-\frac{2 \pi \int_{0}^{R_{K} / \varepsilon} \Phi\left(r^{\prime}\right) r^{\prime} \mathrm{d} r+1}{\left(1+H_{\varepsilon}\left(R_{K}\right)\right)^{1 / 2}\left(1-H_{\varepsilon}\left(R_{K}\right)+2 \mathrm{e}^{2 \gamma} L_{\varepsilon}\left(u, R_{K}\right)^{2}\right)^{2}}\right) .
$$

But $H_{\varepsilon}(0)=L_{\varepsilon}(u, 0)=0, H_{\varepsilon}\left(R_{K}\right)=1+O\left(\varepsilon^{2}\right)$ and $L_{\varepsilon}\left(u, R_{K}\right)=1+O\left(\varepsilon^{2}\right)$ so

$$
\int_{0}^{R_{K}} \tilde{R}_{x y_{\varepsilon}}^{x y} \sqrt{-\tilde{g}_{\varepsilon}} r \mathrm{~d} r=\mathrm{e}^{\gamma}\left(1-\mathrm{e}^{-\gamma}\right)+O(\varepsilon)
$$

We now consider the full four dimensional integral;

$$
\int_{\mathbf{R}^{4}} \tilde{R}_{x y_{\varepsilon}}^{x y} \sqrt{-\tilde{g}_{\varepsilon}} \Psi \mathrm{d} u \mathrm{~d} x \mathrm{~d} y \mathrm{~d} z=I_{3}+I_{4}
$$


where for some $\xi \in[0,1]$,

$$
\begin{aligned}
& I_{3}=\int_{K} \tilde{R}_{x y_{\varepsilon}}^{x y} \sqrt{-\tilde{g}_{\varepsilon}} \Psi(u, 0,0, z) \mathrm{d} u \mathrm{~d} x \mathrm{~d} y \mathrm{~d} z, \\
& I_{4}=\int_{K} \tilde{R}_{x y_{\varepsilon}}^{x y} \sqrt{-\tilde{g}_{\varepsilon}} \frac{\mathrm{d} \Psi}{\mathrm{d} r}(t, \xi x, \xi y, z) r \mathrm{~d} u \mathrm{~d} x \mathrm{~d} y \mathrm{~d} z,
\end{aligned}
$$

Now

$$
\begin{aligned}
\left|I_{4}\right| \leq & M_{7} \int_{B_{\varepsilon}}\left|\tilde{R}_{x y \varepsilon}^{x y} \sqrt{-\tilde{g}_{\varepsilon}}\right| r \mathrm{~d} u \mathrm{~d} x \mathrm{~d} y \mathrm{~d} z \\
& +M_{7} \int_{K-B_{\varepsilon}}\left|\tilde{R}^{x y}{ }_{x y_{\varepsilon}} \sqrt{-\tilde{g}_{\varepsilon}}\right| r \mathrm{~d} u \mathrm{~d} x \mathrm{~d} y \mathrm{~d} z \\
\leq & M_{8} \frac{\left(\varepsilon R_{0}\right)^{3}}{\varepsilon^{2}}+M_{9} \varepsilon^{2}\left(\frac{1}{\varepsilon R_{0}}-\frac{1}{R_{K}}\right)
\end{aligned}
$$

and

$$
I_{3}=\int_{K^{\prime}}\left(2 \pi \mathrm{e}^{\gamma(u)}\left(1-\mathrm{e}^{-\gamma(u)}\right)+O(\varepsilon)\right) \Psi(u, 0,0, z) \mathrm{d} u \mathrm{~d} z
$$

Therefore,

$$
\int_{\mathbf{R}^{4}} \tilde{R}_{x y \varepsilon}^{x y} \sqrt{-\tilde{g}_{\varepsilon}} \Psi \mathrm{d} u \mathrm{~d} x \mathrm{~d} y \mathrm{~d} z=\int_{K^{\prime}} 2 \pi \mathrm{e}^{\gamma(u)}\left(1-\mathrm{e}^{-\gamma(u)}\right) \Psi(u, 0,0, z) \mathrm{d} u \mathrm{~d} z+O(\varepsilon)
$$

and so,

$$
\left[\tilde{R}_{x y}^{x y} \sqrt{-\tilde{g}}\right] \approx 2 \pi \mathrm{e}^{\gamma(u)}\left(1-\mathrm{e}^{-\gamma(u)}\right) \delta^{(2)}(x, y) .
$$

The distributional contributions, at the axis, of the Riemann tensor may be used to calculate the distributional energy-momentum tensor density;

$$
\begin{aligned}
& {\left[\tilde{T}^{u}{ }_{u} \sqrt{-\tilde{g}}\right] \approx T^{u}{ }_{u} \sqrt{-g}-2 \pi \mathrm{e}^{\gamma(u)}\left(1-\mathrm{e}^{-\gamma(u)}\right) \delta^{(2)}(x, y)} \\
& {\left[\tilde{T}^{z}{ }_{z} \sqrt{-\tilde{g}}\right] \approx T^{z}{ }_{z} \sqrt{-g}-2 \pi \mathrm{e}^{\gamma(u)}\left(1-\mathrm{e}^{-\gamma(u)}\right) \delta^{(2)}(x, y)} \\
& {\left[\tilde{T}^{a}{ }_{b} \sqrt{-\tilde{g}}\right] \approx T^{a}{ }_{b} \sqrt{-g} \quad \text { (Other components) }}
\end{aligned}
$$

which still represents a pure radiation solution away from the axis, but whose distributional contributions on the axis are like those of a cosmic string.

A physical interpretation to these contributions may be made in the sense of the mass per unit length of the string: Suppose that $V^{a}$ is a unit time-like vector in the direction of $\partial / \partial t$ and that the distributional and non-distributional matter densities are defined as

$$
\begin{aligned}
& \tilde{\varrho}=\tilde{T}_{a b} V^{a} V^{b} \\
& \varrho=T_{a b} V^{a} V^{b}
\end{aligned}
$$

respectively. We may construct a generalised function of $t$ and $z$, which we shall use to define the mass-per-unit length of the string via weak equivalence, by

$$
\mu(t, z)=\frac{\partial}{\partial z} \int_{C(t, 0, z)}\left[\tilde{\varrho} \sqrt{\tilde{g}^{(3)}}-\varrho \sqrt{g^{(3)}}\right] \mathrm{d} x^{\prime} \mathrm{d} y^{\prime} \mathrm{d} z^{\prime}
$$

where $C(t, a, b)$ is the constant $t$ cylinder $a \leq z \leq b$ of an arbitrary radius and $\tilde{g}^{(3)}$ and $g^{(3)}$ denote that the determinants of the induced metrics on the constant $t$ hypersurfaces.

Here $V^{a}=e^{\gamma} \delta_{u}^{a}$ and

$$
\begin{aligned}
& \tilde{g}^{(3)}=-\mathrm{e}^{-2 \gamma}\left(\frac{1-H_{\varepsilon}+2\left(2 L_{\varepsilon}-1\right) \mathrm{e}^{2 \gamma}}{1-H_{\varepsilon}+2 L_{\varepsilon} \mathrm{e}^{2 \gamma}}\right) \tilde{g}, \\
& g^{(3)}=-\mathrm{e}^{-2 \gamma} g .
\end{aligned}
$$


This will imply that $\sqrt{\tilde{g}^{(3)}} \approx \mathrm{e}^{2 \gamma} \sqrt{-\tilde{g}}$ and hence,

$$
\begin{aligned}
{\left[\tilde{\varrho} \sqrt{\tilde{g}^{(3)}}-\varrho \sqrt{g^{(3)}}\right] \approx } & \mathrm{e}^{-3 \gamma(u)}\left(\tilde{g}_{a u} \tilde{T}^{a}{ }_{u} \sqrt{-\tilde{g}}-g_{a u} T^{a}{ }_{u} \sqrt{-g}\right. \\
\approx & -\mathrm{e}^{-\gamma(u)}\left\{\left(\tilde{T}^{u}{ }_{u}+\frac{x}{r} L_{\varepsilon} \tilde{T}^{x}{ }_{u}+\frac{y}{r} L_{\varepsilon} \tilde{T}^{y}{ }_{u}\right) \sqrt{-\tilde{g}}\right. \\
& \left.-\left(T^{u}{ }_{u}+\frac{x}{r} T^{x}{ }_{u}+\frac{y}{r} T^{y}{ }_{u}\right) \sqrt{-g}\right\} \\
\approx & -\mathrm{e}^{-\gamma(u)}\left\{\tilde{T}^{u}{ }_{u} \sqrt{-\tilde{g}}-T^{u}{ }_{u} \sqrt{-g}+P_{2} L_{\varepsilon}+\gamma^{\prime} / r\right\}
\end{aligned}
$$

The first term will be weakly equivalent to $2 \pi\left(1-\mathrm{e}^{-\gamma(u)}\right) \delta^{(2)}(x, y)$, where as the second term is weakly null, therefore on integrating we obtain

$$
\mu(u, z) \approx 2 \pi\left(1-\mathrm{e}^{-\gamma(u)}\right)
$$

which implies that the string has a mass per unit length of $2 \pi\left(1-\mathrm{e}^{-\gamma(u)}\right)$. This is equal to the angular deficit since $t=u$ at $r=0$.

\section{Asymptopia and conservation of energy}

A natural question to ask is whether or not there is a conservation of energy law. At the local level one usually defines this concept in terms of the contracted Bianchi identities

$$
T^{a}{ }_{b ; a}=0
$$

which certainly hold if the space-time is at least $C^{3}$-regular. If this was interpreted in the context of generalised functions, it would always hold since the smoothed metric is, by definition, $C^{\infty}$.

In the case of radiating space-times, one may formulate the conservation law in a global sense; that is, whether or not there is mass loss between the source, in our case the string at $r=0$, and null infinity ${ }^{(3)} \mathcal{I}$.

In order to enable measurements to be made at null infinity, one must embed the space-time $(M, g)$ into a compact space-time $(\hat{M}, \hat{g})$ whose boundary $\partial M$ represents the points at infinity. In order to preserve causal structure under the embedding, one must impose the condition that $g$ and $\hat{g}$ are conformally related; that is the existence of a smooth function $\Omega$ on $\hat{M}$ such that

$$
\hat{g}_{a b}=\Omega^{2} g_{a b}
$$

and on $\partial M, \Omega=0$ but ${ }^{(3)} \hat{\nabla}_{a} \Omega \neq 0$.

Many formalisms already exist for investigating asymptotic behaviour of four-dimensional spacetimes (Geroch, 1977). These are very often suited to axially-symmetric radiation solutions such as Bondi's radiating metric. A radiating string differs in that the singularity will itself extend out to infinity, thus making a choice of asymptote $(\hat{M}, \hat{g})$ problematic. In the case of our pure radiation solution, which is cylindrically symmetric, we are really measuring the mass-per-unit length of the string and can expect to measure the contribution of this piece of string at infinity. This suggests that a three-dimensional construction of null infinity would be most appropriate.

Recently a formalism for constructing asymptotes at null infinity for cylindrically symmetrical radiating space-times was devised by Ashtekar et al (1996) in which a symmetry reduction to a three-dimensional formalism is performed. This formalism was then used to investigate the asymptotic behaviour of EinsteinRosen waves. Our application of this formalism to our pure radiation solution $(\psi=0, \gamma=\gamma(t-r))$ will be two-fold; firstly, to reveal to what extent it is asymptotically flat, and secondly to calculate the energy flux at null infinity so we can make comparisons with the matter measured on the string by distributional techniques. 


\subsection{Symmetry reduction}

The full four-dimensional metric (1) may be written in the form (Ashtekar et al 1996);

$$
d s^{2}=e^{-2 \psi(3)} g_{a b} d x^{a} d x^{b}+e^{2 \psi} d z^{2}
$$

where the 3-metric may be expressed in terms of Bondi coordinates $(u=t-r, r, \phi)$

$$
d \sigma^{2}={ }^{(3)} g_{a b} d x^{a} d x^{b}=e^{2 \gamma}\left(-d u^{2}-2 d u \mathrm{~d} r\right)+r^{2} d \phi^{2}
$$

The corresponding energy-momentum tensor for the 3-metric will be denoted by ${ }^{(3)} T_{a b}$ and may be calculated from Einstein tensor, via analogous three-dimensional field equations

$$
{ }^{(3)} R_{a b}-\frac{1}{2}{ }^{(3)} R^{(3)} g_{a b}={ }^{(3)} T_{a b}
$$

In general ${ }^{(3)} T_{a b}$ will encode the metric coefficient $\psi$ as a scalar field. (See Ashtekar et al (1997) for Einstein-Rosen waves). In our case we have $\psi=0$ so the only non-zero component of ${ }^{(3)} T_{a b}$ is

$$
{ }^{(3)} T_{u u}=-\frac{\gamma(u)}{r}
$$

In order to examine the structure of null infinity ${ }^{(3)} \mathcal{I}$, we shall need to introduce a conformally related unphysical metric ${ }^{(3)} \hat{g}_{a b}$

$$
d \hat{\sigma}^{2}=\Omega^{2} d \sigma^{2}=e^{2 \gamma(u)}\left(-\hat{r}^{2} d u^{2}+2 d u d \hat{r}\right)+d \phi^{2}
$$

where $\Omega=\hat{r}$ and $\hat{r}=r^{-1}$. Here ${ }^{(3)} \mathcal{I}^{+}$is represented by the points $\hat{r}=0$. Since $\gamma(u)$ is arbitrary and smooth, we automatically have that ${ }^{(3)} \hat{g}_{a b}$ is smooth across ${ }^{(3)} \mathcal{I}^{+}$. Moreover the hypersurface ${ }^{(3)} \mathcal{I}$ will be null; this is because we may define the normal vector $\hat{n}^{a}={ }^{(3)} \hat{g}^{a b\left({ }^{(3)}\right.} \hat{\nabla}_{b} \Omega$, and

$$
{ }^{(3)} \hat{g}_{a b} \hat{n}^{a} \hat{n}^{b}=\hat{r}^{2} e^{-2 \gamma(u)} \rightarrow 0 \quad(\text { as } r \rightarrow 0)
$$

\subsection{Asymptotic flatness}

We now can define asymptotic flatness in the three-dimensional sense. The notation $f \cong g$ will be used to denote that $f$ and $g$ are equal at the points of ${ }^{(3)} \mathcal{I}$.

Definition 1 (Ashtekar et al (1996)). A 3-dimensional space-time $\left(M,{ }^{(3)} g\right)$ is said to be asymptotically flat at null infinity if there is an embedding into a manifold $\left(\hat{M},{ }^{(3)} \hat{g}\right)$ with a smooth metric and a boundary ${ }^{(3)} \mathcal{I}$, topologically $S^{1} \times \mathbf{R}$ such that

(i) $\hat{M}-{ }^{(3)} \mathcal{I}$ is diffeomorphic to $M$.

(ii) ${ }^{(3)} \hat{g}_{a b}=\Omega^{2\left({ }^{(3)}\right.} g_{a b}$ for some smooth function $\Omega$ on $\hat{M}$ such that $\Omega \cong 0$ and ${ }^{\left({ }^{3}\right)} \hat{\nabla}_{a} \Omega \neq 0$.

(iii) $\Omega^{(3)} T_{a b}$ is smooth and $\Omega^{(3)} T_{a b}{ }^{(3)} \hat{g}^{a b} \cong 0$.

(iv) If $\hat{n}^{a}={ }^{(3)} \hat{g}^{a b(3)} \hat{\nabla}_{b} \Omega$ and $\hat{V}^{a}$ is any smooth vector field on $\hat{M}$, tangential to ${ }^{\left({ }^{(3)} \mathcal{I}\right.}$ then $\Omega^{-1{ }^{(3)}} T_{a b} \hat{n}^{a} \hat{V}^{b} \cong$ 0

(v) If ${ }^{(3)} \hat{\nabla}_{a} \hat{n}^{a} \cong 0$ then $\hat{n}^{a}$ is complete on ${ }^{(3)} \mathcal{I}$.

Certainly conditions (i), (ii) and (v) are satisfied by our unphysical metric (3). Condition (iii) is also true since ${ }^{(3)} T_{a b}$ is trace-free and

$$
\Omega^{(3)} T_{u u}=-\hat{r}^{2} \gamma^{\prime}(u) \cong 0
$$

However condition (iv) is not satisfied for our pure radiation solution since

$$
\begin{aligned}
\Omega^{-1(3)} T_{a b} \hat{n}^{a} \hat{V}^{b} & =\hat{r}^{-1(3)} T_{u u}{ }^{(3)} \hat{g}^{u \hat{r}} \hat{V}^{u} \\
& =\gamma^{\prime}(u) e^{-2 \gamma(u)} \hat{V}^{u}
\end{aligned}
$$

The only way to make it vanish would be to force $\gamma$ to be a constant, thus making the string static. However the quantity $\Omega^{-1{ }^{(3)}} T_{a b} \hat{n}^{a} \hat{V}^{b}$ will still be finite and smooth at ${ }^{(3)} \mathcal{I}$. Condition (iv) was introduced in order to guarantee that the energy flux of matter across ${ }^{(3)} \mathcal{I}$ remains finite (Ashtekar et al 1996). We shall show that, despite this condition not being met, one may still define a mass at null infinity. 


\subsection{Bondi mass aspect and mass at null infinity}

Mass at ${ }^{(3)} \mathcal{I}$ may be defined from the energy-momentum tensors. Rather than working with the energymomentum tensors ${ }^{(3)} T_{a b}$ and ${ }^{(3)} \hat{T}_{a b}$ directly, we shall follow Geroch (1977) and Ashtekar et al (1996) and give definitions in terms of the 'modified' energy-momentum tensors

$$
\begin{aligned}
& { }^{(3)} \hat{L}_{a b}=\Omega^{(3)} S_{a b}=\Omega\left({ }^{(3)} R_{a b}-\frac{1}{4}{ }^{(3)} R^{(3)} g_{a b}\right) \\
& { }^{(3)} \hat{S}_{a b}={ }^{(3)} \hat{R}_{a b}-\frac{1}{4}{ }^{(3)} \hat{R}^{(3)} \hat{g}_{a b}
\end{aligned}
$$

The advantage of such tensors is that their algebraic relationship has the fewest number of terms;

$$
{ }^{(3)} \hat{L}_{a b}=\Omega^{\left({ }^{(3)}\right.} \hat{S}_{a b}+{ }^{(3)} \hat{\nabla}_{a} \hat{n}_{b}-\frac{1}{2} \Omega^{-1\left({ }^{(3)}\right.} \hat{g}_{a b}{ }^{(3)} \hat{g}_{c d} \hat{n}^{c} \hat{n}^{d}
$$

We shall denote the pull backs to ${ }^{(3)} \mathcal{I}$ of ${ }^{(3)} \hat{g}_{a b},{ }^{(3)} \hat{L}_{a b}$ and ${ }^{(3)} \hat{S}_{a b}$ as $\underline{g_{a b}}, \underline{L_{a b}}$ and $\underline{S_{a b}}$ respectively. The fact that the pull-back to ${ }^{(3)} \mathcal{I}$ of ${ }^{(3)} \hat{\nabla}_{a} \hat{n}_{b}$ vanishes and that ${ }^{(3)} \mathcal{I}$ is null will be sufficient to guarantee that $g_{a b}$ is a pull-back to ${ }^{(3)} \mathcal{I}$ of a positive definite metric on the space $\mathcal{B}$ of orbits of $\hat{n}^{a}$, which has $S^{1}$ topology, and hence that $\underline{g_{a b}}=\hat{m}_{a} \hat{m}_{b}$, for some 1-form $\hat{m}_{a}$ on ${ }^{(3)} \mathcal{I}$.

If it is the case that for any smooth vector field $\hat{V}^{a},{ }^{\left({ }^{3}\right)} \hat{L}_{a b} \hat{n}^{a} \hat{V}^{b} \cong 0$ and ${ }^{\left({ }^{3}\right)} \hat{S}_{a b} \hat{n}^{a} \hat{V}^{b} \cong 0$ then $\underline{L_{a b}}$ and $\underline{S_{a b}}$ have the form

$$
\underline{L_{a b}}=\underline{L} \hat{m}_{a} \hat{m}_{b}, \quad \underline{S_{a b}}=\underline{S} \hat{m}_{a} \hat{m}_{b}
$$

There are sufficient conditions on the energy momentum tensor which enable ${ }^{(3)} \hat{L}_{a b} \hat{n}^{a} \hat{V}^{b} \cong 0$ and ${ }^{(3)} \hat{S}_{a b} \hat{n}^{a} \hat{V}^{b} \cong 0$ and are weaker than condition (iv) in definition 1 .

Proposition. Suppose that $T \Omega^{-2}, \hat{f}=\Omega^{-2\left({ }^{(3)}\right.} \hat{g}_{a b} \hat{n}^{a} \hat{n}^{b}$ are finite at ${ }^{(3)} \mathcal{I}$ and that for any $\hat{V}^{a}$, tangential to ${ }^{(3)} \mathcal{I},{ }^{(3)} T_{a b} \hat{n}^{a} \hat{V}^{b} \cong 0$ then the quantities ${ }^{(3)} \hat{L}_{a b} \hat{n}^{a} \hat{V}^{b}$ and ${ }^{(3)} \hat{S}_{a b} \hat{n}^{a} \hat{V}^{b}$ both vanish at ${ }^{(3)} \mathcal{I}$

Proof. The vanishing of ${ }^{(3)} \hat{L}_{a b} \hat{n}^{a} \hat{V}^{b}$ is clear from its definition

$$
\begin{aligned}
&{ }^{(3)} \hat{S}_{a b}={ }^{(3)} T_{a b}-\frac{1}{2} T^{(3)} g_{a b} \\
& \Longrightarrow \Omega^{-1\left({ }^{(3)}\right.} \hat{L}_{a b} \hat{n}^{a} \hat{V}^{b}={ }^{(3)} T_{a b} \hat{n}^{a} \hat{V}^{b}-\frac{1}{2} \Omega^{-2} T \hat{n}_{a} \hat{V}^{b} \cong 0 \\
& \Longrightarrow \quad{ }^{(3)} \hat{L}_{a b} \hat{n}^{a} \hat{V}^{b} \cong 0
\end{aligned}
$$

To get ${ }^{(3)} \hat{S}_{a b} \hat{n}^{a} \hat{V}^{b}$ to vanish, we should contract equation (4) with $\hat{n}^{a} \hat{V}^{b}$ to get

$$
{ }^{\left({ }^{3}\right)} \hat{S}_{a b} \hat{n}^{a} \hat{V}^{b}=\Omega^{-1{ }^{(3)}} \hat{L}_{a b} \hat{n}^{a} \hat{V}^{b}-\frac{1}{2} \hat{f} \hat{n}_{a} \hat{V}^{a}-\frac{1}{2} \Omega \hat{V}^{a(3)} \hat{\nabla}_{a} \hat{f}
$$

then apply (5) and the fact that $\hat{f}$ is finite at ${ }^{(3)} \mathcal{I}$.

With the pull-backs in the above forms, we are able to define the Bondi mass aspect as

$$
\hat{B} \hat{m}_{a} \cong(\underline{S}-\underline{L}) \hat{m}_{a}
$$

and if we denote a 1-dimensional cross section of ${ }^{(3)} \mathcal{I}$, to which $\partial / \partial \phi$ is tangential, as $C$ then the mass at ${ }^{(3)} \mathcal{I}$ for this cross section may be defined as

$$
E(C)=\oint(1-\sqrt{2 \hat{B}}) \hat{m}^{a} d S_{a}
$$

So with our solution we obtain a Bondi mass aspect of

$$
\hat{B} \hat{m}_{a} \cong \frac{1}{2} e^{-2 \gamma} \delta_{a}^{\phi}
$$

and the mass at ${ }^{(3)} \mathcal{I}$ for the cross section for $u=$ constant (written as $C_{u}$ ) as

$$
\begin{aligned}
E\left(C_{u}\right) & =\int_{0}^{2 \pi}\left(1-e^{-\gamma(u)}\right) d \phi \\
& =2 \pi\left(1-e^{-\gamma(u)}\right) .
\end{aligned}
$$

Therefore the energy at infinity agrees with the mass per unit length at the axis (2), which implies that there is zero mass loss between the axis and null infinity. 


\section{References}

Ashtekar A, Bičák J and Schmidt B G 1997 Asymptotic structure of symmetry reduced general relativity Phys. Rev. D 55 669-686

Clarke C J S, Vickers J A and Wilson J P 1996 Generalised functions and distributional curvature of cosmic strings Class. Quantum Grav. 13 2485-2498

Colombeau J F 1983 A multiplication of distributions Journal of Mathematical analysis and applications 94 96-115

Colombeau J F 1990 A multiplication of distributions Bulletin of the American Mathematical Society 23 $251-268$

Geroch R P 1977 Asymptotic structure of space-time in Asymptotic structure of space-time ed. F P Esposito and L Witten (Plenum)

Geroch R P and Traschen J 1987 Strings and other distributional sources in general relativity Phys. Rev. D 38 1017-1031

Kramer D, Stephani H, Herlt E and MacCallum M 1980 Exact solutions of Einstein's Field equations (Cambridge University Press)

Krishna Rao J 1964 Cylindrical waves in general relativity Proc. Nat. Sci. India A 30439

Schwartz L 1954 Sur l'impossibilité de la multiplication des distributions. C. R. Acad. Sci. Paris 239 $847-848$ 